\title{
Preparation of trichloroacetoamidoxime in aqueous media and application in one pot synthesis of 1,2,4-oxadiazoles
}

\author{
Lizandra C. Bretanha, ${ }^{\text {a Dalila Venzke, }}$ a Patrick T. Campos, ${ }^{b}$ Anaí Duarte, ${ }^{\text {a }}$ Marcos A. P. \\ Martins, ${ }^{b}$ Geonir M. Siqueira, ${ }^{a} *$ and Rogério A. Freitag ${ }^{a} *$ \\ ${ }^{a}$ Universidade Federal de Pelotas, Departamento de Química e Geociências, 96010-900, \\ Pelotas, RS, Brasil \\ ${ }^{b}$ Departamento de Química, Universidade Federal de Santa Maria, 97105-900 Santa Maria-RS, \\ Brasil \\ E-mail: geonir@ufpel.edu.br, freitag@ufpel.edu.br
}

\begin{abstract}
Oxadiazoles are heterocyclic compounds with a variety application in many pharmaceuticals and agrochemicals products. We reported here the convenient synthesis of 3-trichloromethyl-1,2,4oxadiazoles from trichoroacetoamidoxime and $\mathrm{RC}(\mathrm{O}) \mathrm{Cl}\left(\mathrm{R}=\right.$ methyl ethyl, propyl, $\mathrm{Ph}, \mathrm{CH}_{2} \mathrm{Cl}$, $\mathrm{CHCl}_{2}, \mathrm{CCl}_{3}$ ) by one pot reaction.
\end{abstract}

Keywords: Azoles, 1,2,4-oxadiazoles, heterocycles, $O$-acylation

\section{Introduction}

Azoles and derivatives have a long history in medicinal chemistry. In particular, 1,2,4oxadiazoles represent a class of heterocyclic with a wide variety of biological activities, especially as antiinflamatory, agrochemical and antifungal activities. ${ }^{1-3}$ Biologically relevant compounds containing the 1,2,4-oxadiazole moiety also include HIV integrase inhibitors, ${ }^{4}$ antituberculostatic agents ${ }^{5}$ and antikinetoplastid agents. ${ }^{6}$ In particular, the introduction of halogens and halogenated groups into organic molecules often confers significant changes in their chemical, physical and pharmacological properties. ${ }^{7-10}$

Several methods have been reported in the literature for the synthesis of oxadiazoles. ${ }^{11-15}$ The major synthetic rote to obtain oxadiazoles is the reaction of amidoximes and acyl chloride promoted by either heat or by bases, such $\mathrm{NaH}$, NaOEt or pyridine. Usually this synthesis involve a two step procedure, the first one is the formation of $O$-acyl derivative and the second one and intramolecular cyclization with hard conditions (thionyl chloride, phosphorus oxychloride or acid sulfuric). 
The common protocol for the preparation of precursor amidoximes includes reactions of hydroxylamines with nitriles, or thioamides. ${ }^{16}$ In recent years, we have studied the preparation of halogen-containing building blocks ${ }^{17}$ and have demonstrated their usefulness as precursor on synthesis of five ${ }^{18}$ and six members rings. ${ }^{18-19}$ In according with our program research in organic chemistry, in this work, we chose explore the versatility of the trichloroacetoamidoxime $\mathbf{1}$ as halogen-precursor in a one-pot conversion with acyl chlorides 2a-g to the corresponding 3trichloromethyl-1,2,4-oxadiazoles 3a-g with good yields.

\section{Results and Discussion}

The use of water as a solvent in organic synthesis has been extensively studied, with application in the tetrahydropyranylation of alcohols, Diels-Alder reactions, Claisen Rearrangement, Michael additions, Barbier-Grignard reactions, Reformatsky reactions, enaminations and oxidation and reduction with the research of reaction in water. ${ }^{20-23}$

The precursor trichloroacetoamidoxime $\mathbf{1}$ could be easily synthesized by reaction of trichloroacetonitrile and hydroxylamine hydrochloride in water at room temperature for 3 hours in $90 \%$ yield (Scheme 1 ). This yield was better than the literature (64\%). ${ }^{24}$

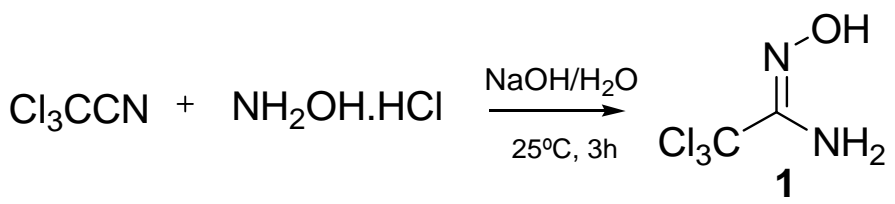

\section{Scheme 1}

In preliminary experiments, we have observed that the reaction between amidoxime $\mathbf{1}$ with trichloroacetyl chloride 2e was solvent and temperature dependent. When the reaction was carried out at low boiling point solvent such as ethyl ether, chloroform or dichloromethane, the starting materials were recovered and we did not observe the formation of the product (entry 1, 2 and 3 - Table 1). In our studies, we found that the toluene was the appropriated solvent for these reactions giving the best results. The increased of temperature raise the formation of 1,2,4oxadiazoles in excellent yield (entry 5 and 6, Table 1). The reaction was monitored by TLC (thin layer chromatography). The important particularity of this reaction process is the work-up, in which we observed that the use of successive treatment with solution of $\mathrm{Na}_{2} \mathrm{CO}_{3}$ gave the products without purifications. 
Table 1. Study of conditions to synthesis of 1,2,4-oxadiazoles 3a-g $\mathbf{g}^{\mathrm{a}}$

\begin{tabular}{|c|c|c|c|c|c|}
\hline Entry & Acyl chloride & Solvent & Temp $\left({ }^{\circ} \mathrm{C}\right)$ & Product & Yield (\%) \\
\hline 1 & $2 e$ & Ethyl ether & 34 & & - \\
\hline 2 & $\mathrm{Cl}_{3} \mathrm{C}_{2 \mathrm{e}} \mathrm{Cl}$ & $\mathrm{CH}_{2} \mathrm{Cl}_{2}$ & 40 & & - \\
\hline 3 & $\mathrm{Cl}_{3} \mathrm{C}_{2 \mathbf{e}}$ & $\mathrm{CHCl}_{3}$ & 61 & & - \\
\hline 4 & $\mathrm{Cl}_{3} \mathrm{C}_{2 \mathrm{e}}$ & Solvent free & 100 & & - \\
\hline 5 & $\mathrm{Cl}_{3} \mathrm{C}_{2 \mathrm{e}}$ & Toluene & 100 & & 90 \\
\hline 6 & $2 \mathrm{Cl}$ & Toluene & 100 & & 88 \\
\hline
\end{tabular}

${ }^{\mathrm{a}}$ reaction time $20 \mathrm{~h}$.

The 5-substituted-3-trichloromethyl-1,2,4-oxadiazoles 3a-g were synthesized by treatment of trichloroacetiamidoxime $\mathbf{1}$ with acyl chlorides 2a-g using toluene for 20 hours at $100^{\circ} \mathrm{C}$ (Scheme 2). The compounds 3a-g were obtained in good yields (60-90\%). The scope and generality of this process is illustrated by a series of seven oxadiazoles and the results are presented in Table 2.<smiles>N/C(=N/O)C(Cl)(Cl)Cl</smiles>

1

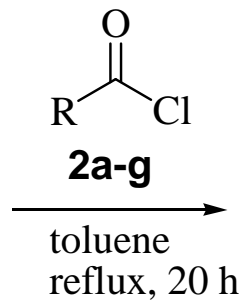<smiles>[R]c1nc(C(Cl)(Cl)Cl)no1</smiles>

3a-g

\section{Scheme 2}


Table 2. Synthesis of 3 trichloromethyl-1, 2, 4 oxadiazoles 3a-g

(1)

a yield of isolated compound

The products were identified concerning both analytical and spectral data $\left({ }^{1} \mathrm{H}\right.$ NMR and ${ }^{13} \mathrm{C}$ NMR) of all compounds are in full agreement with the proposed structure.

\section{Conclusions}

In conclusion, we have described the facile preparation of trichloroacetoamidoxime $\mathbf{1}$ in aqueous media, and its application in an one-pot approach to the synthesis of 1,2,4-oxadiazoles in good yields. This method works well with a variety of acyl chlorides. 


\section{Experimental Section}

General. Starting trichloroacetoamidoxime 1 was prepared from of our procedure. All solvents and reagents were obtained from Aldrich and used without further purification. The data of NMR spectra were recorded on a Bruker DPX $400\left({ }^{1} \mathrm{H}\right.$ at $400.13 \mathrm{MHz}$ and for ${ }^{13} \mathrm{C}$ at $100.63 \mathrm{MHz}$ ) spectrometer, $5 \mathrm{~mm}$ sample tubs, $298 \mathrm{~K}$, digital resolution of $\pm 0.01 \mathrm{ppm}, 0,5 \mathrm{M}$ in $\mathrm{CDCl}_{3}$, containing TMS as internal standard. Mass spectra were registered in HP 6890 GC connected to HP 5973 MSD and interfaced by a Pentium PC. The CG was equipped with a split-splitless injector, autosampler, cross-linked HP-5 capillary column (30m, 0,32 mm of internal diameter), and helium was used as the carrier gas.

\section{General procedures. Trichloroacetoamidoxime (1)}

A mixture of hydroxylamine hydrochloride $2.36 \mathrm{~g}(3.5 \mathrm{mmol})$ and $\mathrm{NaOH} 1.36 \mathrm{~g}(3.5 \mathrm{mmol})$ in water was added trichloroacetonitrile $1.7 \mathrm{~mL}(1.7 \mathrm{mmol})$. The mixture was stirred at room temperature for $3 \mathrm{~h}$. For the compound 1 a solid was observed. The product was isolated by filtered, dried in a vacuum and recrystalized in gradient hexane/acetate (3:1).

\section{Synthesis of 1,2,4-oxadiazoles 3a-g}

A mixture of acyl chloride $\mathbf{2 a - g}(6 \mathrm{mmol})$ in toluene was added dropwise in a solution of the trichloroacetoamidoxime 1 (3 mmol) in toluene at $0{ }^{\circ} \mathrm{C}$. The reaction mixture was stirred for $20 \mathrm{~h}$ at reflux. After the time the solvent was remove and $\mathrm{CH}_{2} \mathrm{Cl}_{2}$ was added, the organic solution was washed (twice in water), (twice in solution $\mathrm{Na}_{2} \mathrm{CO}_{3}$ ), and (once in water). The organic layer was dried $\left(\mathrm{Na}_{2} \mathrm{SO}_{4}\right)$ and the solvent removed under pressure. Finally, the 1, 2, 4-oxadiazoles 3a-g were obtained in good yields.

3-Trichloromethyl-5-methyl-1,2,4-oxadiazole (3a). $\mathrm{C}_{4} \mathrm{H}_{3} \mathrm{Cl}_{3} \mathrm{~N}_{2} \mathrm{O}$; 201.5; mp $54^{\circ} \mathrm{C}$; bp $203.7^{\circ} \mathrm{C}$; yield (61\%); ${ }^{1} \mathrm{H}$ NMR (400 MHz; $\left.\mathrm{CDCl}_{3}\right): \delta(\mathrm{ppm}) 2.70\left(3 \mathrm{H}, \mathrm{s}, \mathrm{CH}_{3}\right) ;{ }^{13} \mathrm{C} \mathrm{NMR}(100 \mathrm{MHz}$; $\left.\mathrm{CDCl}_{3}\right): \delta(\mathrm{ppm})$ 179.2, 170.9; $99.96\left(\mathrm{CCl}_{3}\right) ; 12.4\left(\mathrm{CH}_{3}\right)$; MS CG-MS (EI, $\left.70 \mathrm{eV}\right): \mathrm{m} / \mathrm{z} 200\left(\mathrm{M}^{+}\right.$1, 1), 169 (11.4), 167(61.9) 165(100), 131(9.5), 129(9.5), 126(15.2), 124(19);

3-Trichloromethyl-5-ethyl-1,2,4-oxadiazole (3b). $\mathrm{C}_{5} \mathrm{H}_{5} \mathrm{Cl}_{3} \mathrm{~N}_{2} \mathrm{O} ; 215.5$; oil; yield (60\%); ${ }^{1} \mathrm{H}$ NMR (400 MHz; $\left.\mathrm{CDCl}_{3}\right): \delta(\mathrm{ppm}) 3.03\left(2 \mathrm{H}, \mathrm{q}, \mathrm{CH}_{2}\right) ; 1.50\left(3 \mathrm{H}, \mathrm{t}, \mathrm{CH}_{3}\right) ;{ }^{13} \mathrm{C} \mathrm{NMR}(100 \mathrm{MHz}$; $\left.\mathrm{CDCl}_{3}\right): \delta(\mathrm{ppm})$ 170.8, 183.3; $109.5\left(\mathrm{CCl}_{3}\right) ; 20.5\left(\mathrm{CH}_{2}\right) ; 10.4\left(\mathrm{CH}_{3}\right) ; \mathrm{MS}$ CG-MS (EI $\left.70 \mathrm{eV}\right)$ : $\mathrm{m} / \mathrm{z} 214$ ( $\left.\mathrm{M}^{+}-1,1\right), 182.9(10), 180.9(60.7), 179.9(6), 178.9(100), 126(24), 124(38) ;$

3-Trichloromethyl-5-propyl-1,2,4-oxadiazole (3c). $\mathrm{C}_{6} \mathrm{H}_{7} \mathrm{Cl}_{3} \mathrm{~N}_{2} \mathrm{O}$; 229.5; oil; yield (74\%); ${ }^{1} \mathrm{H}$ NMR (400 MHz; $\left.\mathrm{CDCl}_{3}\right): \delta(\mathrm{ppm}) 2.48\left(2 \mathrm{H}, \mathrm{t}, \mathrm{CH}_{2}\right) ; 1.59\left(2 \mathrm{H}, \mathrm{m}, \mathrm{CH}_{2}\right) ; 0.85\left(3 \mathrm{H}, \mathrm{t}, \mathrm{CH}_{3}\right) ;{ }^{13} \mathrm{C}$ NMR (100 MHz; $\left.\mathrm{CDCl}_{3}\right): \delta(\mathrm{ppm})$ 171.1, 178.8; $104.8\left(\mathrm{CCl}_{3}\right) ; 29.7\left(\mathrm{CH}_{2}\right) ; 20.6\left(\mathrm{CH}_{2}\right) ; 13.3$ $\left(\mathrm{CH}_{3}\right) ; 227$ (M+2, 11); 213 (1.2); 199(5.7); 185(6); 171(6); 143(17); 129(8.7); 101(8); 97(9.4); 88(6.8); 87(70); 84(5); 83(12); 81(5); 75(17); 74(100); 71(7); 69(17); 67(5); 59(8); 57(16); 43(27)

3-Trichloromethyl-5-phenyl-1,2,4-oxadiazole (3d) $\mathrm{C}_{9} \mathrm{H}_{5} \mathrm{Cl}_{3} \mathrm{~N}_{2} \mathrm{O}$; 263.5; mp 65 and bp $106{ }^{\circ} \mathrm{C}$; yield (88\%); ${ }^{1} \mathrm{H}$ NMR (400 MHz; $\mathrm{CDCl}_{3}$ ): $\delta$ (ppm) 8.2 and 7.5 (m, Ph); MS CG-MS (EI $70 \mathrm{eV}$ ): 
m/z (\%) 263, 262, 264 (9)[M+] 229(63), 228(9.5), 227(100), 126(22), 124(33), 105(25), 103(35), 77(74), 76(21), 50(21), 51(43

3,5-Bis-trichloromethyl-1,2,4-oxadiazole (3e). $\mathrm{C}_{4} \mathrm{Cl}_{6} \mathrm{~N}_{2} \mathrm{O}$; 305; bp $250{ }^{\circ} \mathrm{C}$; yield (90\%); ${ }^{13} \mathrm{C}$ NMR (100 MHz; CDCl 3 ): $\delta(\mathrm{ppm})$ 190.5, 195.5; $103.8\left(\mathrm{CCl}_{3}\right)$; $101.6\left(\mathrm{CCl}_{3}\right)$; MS CG-MS (EI 70 $\mathrm{eV}): \mathrm{m} / \mathrm{z}$ (\%) $304\left(\mathrm{M}^{+}-1,1\right), 274.5(4), 273(20), 271(62.8), 268.9(100), 267(64.8), 206(3.8)$, 203.9(7.6), 202(4.8), 128.9(2.9), 126(4.8), 124(4.8), 119(11.4), 116.9(13.3), 100(4.8), 98(6.7), 94(2.8), 84(6.7), 82(9.5), 73(1.9), 47(4.8).

5-Dichloromethyl-3-trichloromethyl-1,2,4-oxadiazole (3f). $\mathrm{C}_{4} \mathrm{HCl}_{5} \mathrm{~N}_{2} \mathrm{O}$; 270.5; bp $253{ }^{\circ} \mathrm{C}$; yield (71\%); ${ }^{1} \mathrm{H}$ NMR (400 MHz; $\left.\mathrm{CDCl}_{3}\right): \delta(\mathrm{ppm}) 6.8$ (H, s, CH); MS CG-MS (EI $\left.70 \mathrm{eV}\right): \mathrm{m} / \mathrm{z}$ (\%) $270\left(\mathrm{M}^{+}, 10\right), 272.8(21), 271.8(5), 269.9(5), 268.8(100), 266.9(65), 236.9(5), 234.9(8)$, 232.9(6), 205.8(4), 203.85(8.5), 201.8(6.5), 126(7), 124(11), 121(6), 119(19), 117(23), 110(11), 108(14), 83(15), 81.9(24), 49(6), 47(17);

5-Chloromethyl-3-trichloromethyl-1,2,4-oxadiazole (3g). $\mathrm{C}_{4} \mathrm{H}_{2} \mathrm{Cl}_{4} \mathrm{~N}_{2} \mathrm{O}$; 235; bp 242; yield (70\%); ${ }^{1} \mathrm{H}$ NMR (400 MHz; $\left.\mathrm{CDCl}_{3}\right): \delta$ (ppm) 4,7 (2H, s, $\left.\mathrm{CH}_{2}\right)$ ); ${ }^{13} \mathrm{C}$ NMR (100 MHz; $\left.\mathrm{CDCl}_{3}\right): \delta$ (ppm) 176.8, 171.3; $109.5\left(\mathrm{CCl}_{3}\right)$; $33.06\left(\mathrm{CH}_{2}\right)$; MS CG-MS (EI $\left.70 \mathrm{eV}\right): \mathrm{m} / \mathrm{z}(\%) 237\left(\mathrm{M}^{+}+2\right.$, 45), 235(100), 233.9(5), 232.9(89), 126(8), 124(13), 118(4), 116.9(5), 82.9(12), 81.9(5);

\section{Acknowledgements}

The authors are grateful to FAPERGS, CNPq (Project 476769/2004-3) and CAPES for financial support. Authors also thank to Professor Claudio M.P Pereira (UFPel) and Professor Wilson Cunico (UFPel) for the valuable contributions on the preparation of this manuscript.

\section{References}

1. Cesarini, S.; Colombo, N.; Pulici, M.; Felder, E. R.; Brill, W. Tetrahedon 2006, 62, 10223.

2. Kangani, C. O.; Kelley, D. E.; Day, B. W Tetrahedron Lett. 2006, 47, 6497.

3. Polshettiwar, V.; Varma, R. S. Tetrahedron Lett. 2008, 49, 879.

4. Rajapakse, H. A.; Zhu, H.; Young, M. B.; Mott, B. T. Tetrahedron Lett. 2006, 47, 4827.

5. Vazquez, G. N.; Salinas, G. M. M.; Fajardo, Z. V. D.; Villarreal, J. V.; Soto, S. E.; Salazar, F. G.; Nunes, E. H.; Fernandez, S. S. Bioorg. Med. Chem. 2007, 15, 5502.

6. Cottrell, D. M.; Capers, J.; Salem, M. M.; Fradley, K. D. L.; Croft, S. L.; Werbovetz, K. A. Bioorg Med. Chem. 2004, 12, 2815.

7. Karthikeyan, M. S.; Prasad, D. J.; Mahalinga. M.; Holla, B. S.; Kumari, N. S. Eur. J. Med. Chem. 2008, 43, 25.

8. Pereira, C. M. P.; Quina, F. H.; Silva, F. A. N.; Emmerich, D. J.; Machulek Jr, A. Mini-Rev. Org. Chem. 2008, 5, 331.

9. Druzhinin, S. V.; Balenkova, E. S.; Nenajdenko, V. D. Tetrahedron 2007, 63, 7753. 
10. Bonacorso, H. G.; Lewandowski, H.; Drekener, R. L.; Costa, M. B.; Pereira, C. M. P.; Wastowski, A. D.; Peppe, C.; Martins, M. A. P.; Zanata, N. J. Fluorine Chem. 2003, 122, 159.

11. Eloy, F.; Lenaers, R.; Moussebois, C. U.S. Patent 3227 725, 1966.

12. Gutman, A. D. U.S. Patent 4279 638, 1981.

13. (a) Eloy, F.; Lenaers, R.; Buyle, Bull. Soc. Chim. Belg. 1964, 73, 518. (b) Lenaers, R. U.S. Patent 3211742, $19651012,1965$.

14. Katritzky, A. R.; Shestopalov, A. A.; Suzuki, K. Arkivoc 2005, (vii), 36.

15. Liang, G. B.; Feng, D. D. Tetrahedron Lett. 1996, 37, 662.

16. Katritzky, A. R.; Khashad, N. M.; Kirichenko, N.; Singh, A. J. Org. Chem. 2006, 71, 9051.

17. Martins, M. A. P.; Cunico, W.; Pereira, C. M. P.; Sinhorin, A. P.; Flores, A. C. F.; Bonacorso, H. G.; Zanatta, N. Curr. Org. Synth. 2004, 1, 391.

18. (a) Martins, M. A. P; Cunico, W.; Brondani, S.; Peres, R. L.; Zimmermann, N.; Rosa, F. A.; Fiss, G. F.; Zanatta, N.; Bonacorso, H. G. Synthesis 2006, 1485. (b) Pereira, C. M. P.; Stefani, H. A.; Guzen, K. P.; Órfão, A. T. G. Lett. Org. Chem. 2007, 4, 43. (c) Martins, M. A. P.; Freitag, R. A.; Da Rosa, A.; Flores, A. F. C.; Zanatta, N.; Bonacorso, H. G. J. Heterocyclic Chem. 1999, 36, 217. (d) Martins, M. A. P.; Sinhorin, A. P.; Da Rosa, A.; Flores, A. F. C.; Wastowski, A. D.; Pereira, C. M. P.; Flores, D. C.; Beck, P.; Freitag, R. A.; Brondani, S.; Cunico, W.; Bonacorso, H. G.; Zanatta, N. Synthesis 2002, 16, 2353.

19. Martins, M. A. P.; Cunico, W.; Scapin, E.; Emmerich, D. J.; Fiss, G. F.; Rosa, F. A.; Bonacorso, H. G.; Zanatta, N.; Flores, A. F. C. Lett. Org. Chem. 2006, 3, 358.

20. Stefani, H. A.; Pereira, C. M. P.; Dörr, F. A.; Cella, R. Arkivoc 2005, (vi), 19.

21. Chanda, A.; Fokin, V. V. Chem Rev. 2009, 109, 725

22. Amantini, D.; Fringelli, F.; Piermatti, O.; Tortoioli, S.; Vaccaro, L. Arkivoc 2002, (xi), 293

23. (a) Tundo, P.; Anastas, Green Chemistry: Challenging Perspectives Eds. P. T. Oxford Sc. Pub: 1999. (b) Lester, T. Chem. Brit. 1996, 45.

24. Hynes, J. B.; Hack, L. G. J. Med. Chem. 1972, 15, 1194. 